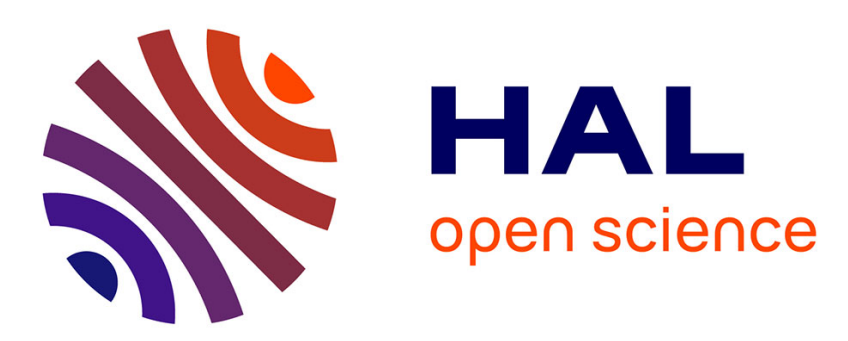

\title{
Comment on "Nano-particle magnetism with a dispersion of particle sizes" [J. Appl. Phys. 112, 103915 (2012)]
}

Florent Tournus, Alexandre Tamion

\section{- To cite this version:}

Florent Tournus, Alexandre Tamion. Comment on "Nano-particle magnetism with a dispersion of particle sizes" [J. Appl. Phys. 112, 103915 (2012)]. Journal of Applied Physics, 2013, 114 (12), pp.126101. 10.1063/1.4823813 . hal-03040018

\section{HAL Id: hal-03040018 \\ https://hal.science/hal-03040018}

Submitted on 4 Dec 2020

HAL is a multi-disciplinary open access archive for the deposit and dissemination of scientific research documents, whether they are published or not. The documents may come from teaching and research institutions in France or abroad, or from public or private research centers.
L'archive ouverte pluridisciplinaire HAL, est destinée au dépôt et à la diffusion de documents scientifiques de niveau recherche, publiés ou non, émanant des établissements d'enseignement et de recherche français ou étrangers, des laboratoires publics ou privés. 


\section{Comment on "Nano-particle magnetism with a dispersion of particle sizes" []. Appl. Phys. 112,103915 (2012)]}

Cite as: J. Appl. Phys. 114, 126101 (2013); https://doi.org/10.1063/1.4823813

Submitted: 06 September 2013 . Accepted: 13 September 2013 . Published Online: 27 September 2013

F. Tournus, and A. Tamion
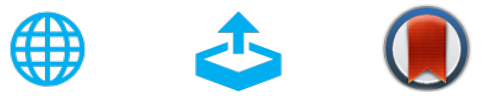

View Online

Export Citation

CrossMark

\section{ARTICLES YOU MAY BE INTERESTED IN}

Nano-particle magnetism with a dispersion of particle sizes

Journal of Applied Physics 112, 103915 (2012); https://doi.org/10.1063/1.4766817

Accurate determination of the magnetic anisotropy in cluster-assembled nanostructures

Applied Physics Letters 95, 062503 (2009); https://doi.org/10.1063/1.3200950

Determination of the blocking temperature of magnetic nanoparticles: The good, the bad, and the ugly

Journal of Applied Physics 118, 184304 (2015); https://doi.org/10.1063/1.4935484

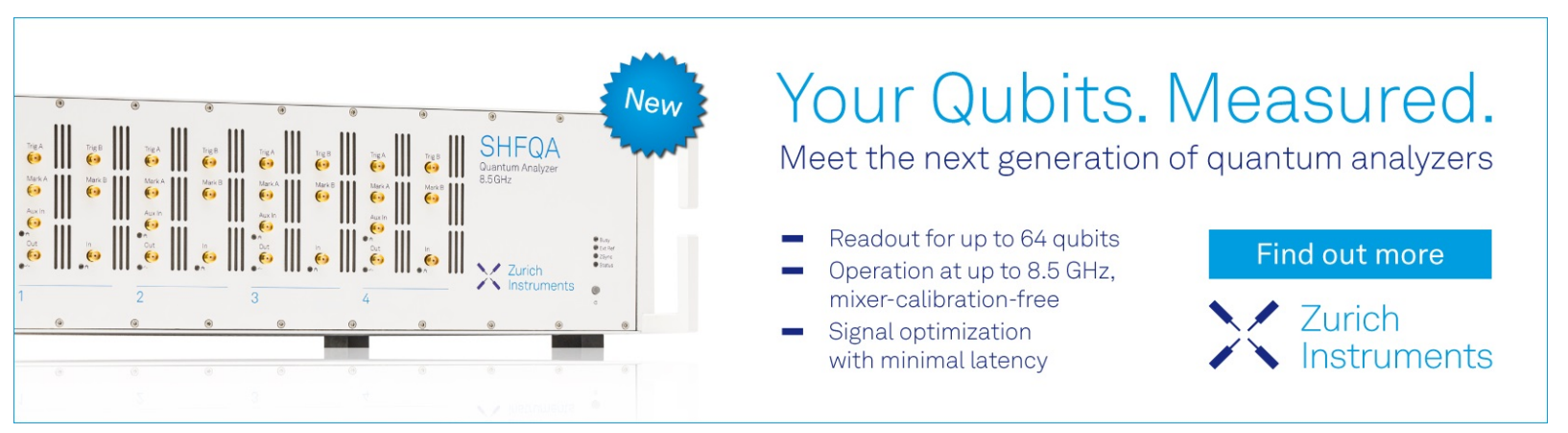




\title{
Comment on "Nano-particle magnetism with a dispersion of particle sizes" [J. Appl. Phys. 112, 103915 (2012)]
}

\author{
F. Tournus ${ }^{\text {a) }}$ and A. Tamion \\ Institut Lumière Matière, UMR5306 Université Lyon 1-CNRS, Université de Lyon, 69622 Villeurbanne cedex, \\ France
}

(Received 6 September 2013; accepted 13 September 2013; published online 27 September 2013)

\begin{abstract}
A recent paper $^{1}$ examines zero field-cooled/field-cooled (ZFC/FC) susceptibility curves for nanoparticle assemblies with a size distribution. It is explained that the "volume and number weighted distribution are equally valid for the representation of distribution functions in nanoparticle magnetic systems" and the usual modelling approach (abrupt transition from a blocked to a superparamagnetic regime, at a given temperature) is compared to the more elaborate one (the "progressive crossover model (PCM)") introduced in our previous articles. ${ }^{2-4}$ The importance of the $f_{0}$ value is also stressed. In this article, several statements are made in opposition to some of our previously published results. Because we like to believe that these words were driven by a simple "misunderstanding" of our models and analysis, we would like to clarify some points in the present comment. C 2013 AIP Publishing LLC. [http://dx.doi.org/10.1063/1.4823813]
\end{abstract}

\section{DISCRIMINATION OF PARTICLE SIZE DISTRIBUTIONS WITH A SUPERPARAMAGNETIC MAGNETIZATION LOOP}

Based on his own numerical calculations shown in Fig. 2 of Ref. 1, the author states that his results "are in disagreement" with ours. He adds "the Tamion et al. finding that the magnetization curves are not sensitive to the particle size distribution parameters is not correct" and "any analysis of the room temperature magnetization curve should clearly and unambiguously provide information concerning the particle size distribution." Unfortunately, Fig. 2 cannot be compared to our results in Ref. 4 since the author of Ref. 1 only modifies the median diameter $D_{m}$ of the distribution keeping the standard deviation $\sigma_{D}$ constant, while we had changed these two parameters jointly. Of course, if one parameter is modified, the magnetization curve will not be the same. Moreover, the ability of a superparamagnetic magnetization loop to discriminate different particle size distribution had been further discussed in our response ${ }^{5}$ to a comment ${ }^{6}$ of our paper (Ref. 10 in Ref. 1). We demonstrated that the superparamagnetic loop alone is much less sensitive to the details of the size distribution than zero field-cooled/field-cooled (ZFC/FC) curves, that the best fit of a superparamagnetic loop alone will not give the same result as with the "triple fit" procedure and that, as shown here in Fig. 1, the room temperature magnetization loop will definitely not provide unambiguous information (that is, several particle distributions can correctly fit the experimental curve).

\section{PARTICLE SIZE DISTRIBUTION AND PROBABILITY DENSITY FUNCTION (PDF)}

In Ref. 1, it is written that our supposed "failure to recognize the different definitions for the volume and number weighted distribution functions" led us to "mistaken

${ }^{a)}$ Electronic mail: florent.tournus@univ-lyon1.fr conclusions like stating that the equation for the initial susceptibility in Refs. 14-16 is erroneous." This has already been discussed in a previous paper by El-Hilo and Chantrell. ${ }^{7}$ It is possible to use either the particle size distribution $\rho(V)$ or the "volume weighted distribution" $f(V)$ [or with the diameter as the variable, $\rho(D)$ and $f(D)]$. The number of particles having a diameter in the interval $[D ; D+d D]$ is given by $N(D, D+d D)=N_{\text {tot }} \rho(D) d D$, while $f(D) d D$ represents the volume fraction corresponding to particles having a diameter in the interval $[D ; D+d D]$. This means that only $\rho(D)$ is the PDF of the particle diameter. This is a mathematical definition and not just a question of usage: ${ }^{8}$ for a given random variable, there is only one PDF. It is then incorrect to state ${ }^{1}$ "four types of probability density functions are frequently used when dealing with particle size distributions in magnetic nanoparticles systems."

We perfectly agree that the two formulations using either the particle size distribution $\rho(V)$ or the "volume weighted distribution" $f(V)$ are equivalent ${ }^{7}$ [it can be seen directly by setting $f(V)=V \rho(V) / \bar{V}]$. Unfortunately, the fact is that many authors ${ }^{9}$ did not notice in the original paper ${ }^{10}$ the important difference between $f(V)$ and the particle size distribution $\rho(V)$. The consequence is an erroneous interpretation of experimental data, and this was what we wanted to point out in our article. ${ }^{3}$ To make it short, it was not the formula which was erroneous, but its (frequent) usage. Therefore, pointing towards a "frequent error met in the literature" was not a misconception. We believe that the choice of $\rho(D)$ (the "number distribution") is the most judicious: $\rho(D)$ is the particle size probability density function (which then allows easy computation of mean values ${ }^{11}$ ), it is much more familiar to experimentalists because it is usually accessible from observations, and in fact we did not find in the literature any other studies using the original expression of Ref. 10 together with the volume fraction definition of $f(y)$. Furthermore, a lognormal size distribution is often experimentally observed: this justifies the common assumption that 


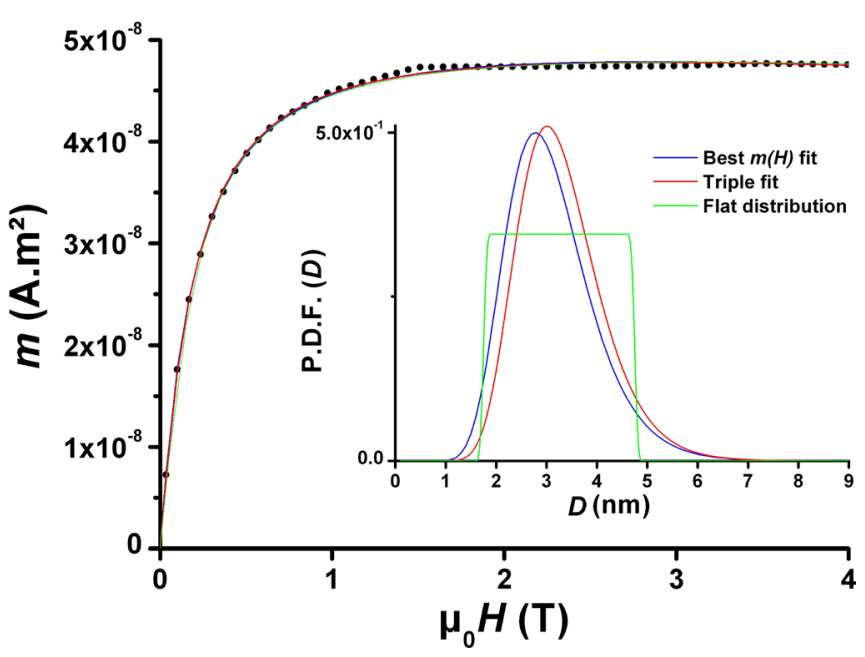

FIG. 1. Superparamagnetic $m(H)$ magnetization curves calculated for different particle size distribution (shown in insert: lognormal distribution corresponding to the best $m(H)$ fit, lognormal distribution corresponding to the best "triple fit," and unphysical flat distribution), compared to an experimental curve (points). This demonstrates the poor ability of a superparamagnetic loop alone to discriminate between different particle size distributions (the corresponding $\mathrm{ZFC/FC}$ curves would be very different).

$\rho(D)$ is a lognormal; but considering the volume fraction, we do not see any particular reason for $f(D)$ to be lognormal.

\section{PROGRESSIVE CROSSOVER MODEL}

In Ref. 1, the ZFC analytical expression that we had established in Ref. 2 is used, but the author erroneously considers a constant "measurement time" $t_{m}$. In Ref. 2 , it was clearly explained that a measurement at a constant temperature sweeping rate $r_{T}$ corresponds to an "effective waiting time" $\delta_{t}$, or $t_{m}$ with the notation of Ref. 1 , which varies with the temperature and the magnetic anisotropy energy (MAE) $K=K_{\text {eff }} V$ (i.e., with the particle volume $V$ ). We have thus established

$$
t_{m} \simeq 0.6727 \frac{T}{r_{T}}\left(\frac{k_{B} T}{K}\right)^{0.9}
$$

The purpose of our derivation of an (approximate) analytical expression of ZFC/FC susceptibility curves ${ }^{2}$ was to consider the situation of a temperature sweep as in the experiments, in order to obtain an improved description compared to the "abrupt transition model" (ATM). Therefore, using a $\exp \left(-t_{m} / \tau\right)$ term with a constant $t_{m}$ is not at all our "PCM." Simulated curves of Ref. 1 with a constant $t_{m}=1 \mathrm{~s}$ (in particular Figs. 4 and 5 of Ref. 1) do not correspond to any experimental situation $^{12}$ and thus cannot be compared to our simulations where $t_{m}$ varies along the curves $\left(t_{m}\right.$ dependence on $T)$ and among the particles in an assembly $\left(t_{m}\right.$ dependence on $K=K_{\text {eff }} V$ ).

Furthermore, stating that ${ }^{1}$ "defining new transition crossover temperatures like $T_{X}$, in order to improve the critical approach has no physical meaning" does only reflect the misunderstanding of our previous article. Our proposition of an "improved two-state model" 3 stems from the fact that $t_{m}$ is not constant. As it can be seen on Figs. 3 and 4 of Ref. 3, using a carefully defined transition temperature $T_{X}$ rather than the usual $T_{B}$ expression (where the temperature sweeping rate is not considered at all) results in different curves and appears to be necessary to get a good agreement with the "exact" curves (in particular for the FC at low temperature). This "improved two-state model" is then not only physically sound, but also useful from a practical point of view. We had also shown ${ }^{3}$ that for broad enough MAE distributions (most of the realistic cases), this two-state model is equivalent to the PCM, what is stressed again in Ref. 1.

\section{IV. "BLOCKING" TEMPERATURE DISTRIBUTION}

In Ref. 1, the author claims that Eqs. (22) and (23), relating the "blocking" or "transition" temperature distribution to the $F C-Z F C$ difference, are both valid but simply give different physical quantities. However, there is only one PDF for the transition temperature and it corresponds to the $F_{N}(T)$ of Ref. 1: this is what anyone would call the "blocking temperature distribution." The other distribution $F_{V}(T)$ would be a "volume fraction distribution of the blocking temperature," which is not trivial and can lead to some confusions. ${ }^{13}$ For example, some authors (Refs. 23-25 of Ref. 3) have used Eq. (22) to derive a distribution which they call the "blocking temperature distribution," what is in fact erroneous. (Sometimes, this distribution is then compared to the particle volume distribution, not the volume fraction distribution.)

\section{INFLUENCE OF $\boldsymbol{f}_{0}$}

At the end of Ref. 1, it is written that one of our result from Ref. 3 is "obviously incorrect." We had shown that two different sets of parameters can result in almost the same ZFC/FC curves (see Fig. 1 of Ref. 3): with the conditions considered, $f_{0}=10^{9} \mathrm{~Hz}$ and $K_{\text {eff }}=100 \mathrm{~kJ} / \mathrm{m}^{3}$ gives $\mathrm{ZFC} / \mathrm{FC}$ curves very similar to $f_{0}=10^{6} \mathrm{~Hz}$ and $K_{\text {eff }}=72.5 \mathrm{~kJ} / \mathrm{m}^{3}$. With the "critical approach," i.e., the abrupt transition model, the author of Ref. 1 does not find the same conclusion: the two different sets of parameters correspond to slightly different transition temperatures. This is perfectly normal, since in our case, we use the more elaborate progressive crossover model (to reproduce an experimental ZFC/FC measurement), where as reminded above the effective measurement time $t_{m}$ depends on several parameters and in particular the magnetic anisotropy constant $K_{\text {eff }}$. This is in contrast with the situation examined in Ref. 1 where $t_{m}$ was fixed to a constant value. Therefore, it cannot be stated in Ref. 1 "the exact $\chi(T)$ variation for the two sets of values was re-calculated, and the obtained data (.) clearly show that the two variations are not identical." Anyone can verify, using the formula given in our previous articles, ${ }^{2,3}$ not by mixing different models like Ref. 1 did, that our conclusions are correct.

It is also surprising to read in Ref. 1 "Another confusing result in Ref. 12 is the effect of $f_{0}$ on the $\chi(T)$ variation, which was considered not to be an important issue," whereas the influence of $f_{0}$ had been examined in our articles ${ }^{2,3}$ (the $f_{0}$ dependence is explicitly given in the expression of $T_{B}$ and $T_{X}$ for instance) and we had written "many couples of $f_{0}$ and 
$K_{\text {eff }}$ values can provide a good fit: the lack of information on $f_{0}$ thus results in an uncertainty on $K_{\text {eff }}$. The indetermination existing for ZFC/FC curves can be removed, at least partly, by adjusting simultaneously different magnetization curves sharing common parameters."

\section{CONCLUSION}

The doubt that Ref. 1 tries to shed on our previous conclusions only comes from a misunderstanding of some of our published results. The categoric statements of the author of Ref. 1 are based on calculations which are in fact not comparable to what we did, even if they look similar (in particular, we insist on the fact that the author makes a bad usage of the ZFC analytical formula that we have derived, because he considers a constant $t_{m}$ ). Finally, a careful examination of our previous articles ${ }^{2-5}$ will give a clearer view on the points discussed in this comment.

${ }^{1}$ M. El-Hilo, J. Appl. Phys. 112, 103915 (2012).

${ }^{2}$ F. Tournus and E. Bonet, J. Magn. Magn. Mater. 323, 1109 (2011).

${ }^{3}$ F. Tournus and A. Tamion, J. Magn. Magn. Mater. 323, 1118 (2011).

${ }^{4}$ A. Tamion, M. Hillenkamp, F. Tournus, E. Bonet, and V. Dupuis, Appl. Phys. Lett. 95, 062503 (2009).

${ }^{5}$ A. Tamion, M. Hillenkamp, F. Tournus, E. Bonet, and V. Dupuis, Appl. Phys. Lett. 100, 136102 (2012).
${ }^{6}$ P. S. Normile and J. A. De Toro, Appl. Phys. Lett. 100, 136101 (2012). ${ }^{7}$ M. El-Hilo and R. W. Chantrell, J. Magn. Magn. Mater. 324, 2593 (2012).

${ }^{8}$ The probability $P\left(D_{0}<D<D_{0}+d D_{0}\right)$ to be in a given interval is given by the ratio $N\left(D_{0}, D_{0}+d D_{0}\right) / N_{\text {tot }}$ and corresponds to $\operatorname{PDF}\left(D_{0}\right) d D_{0}$. See for instance http://mathworld.wolfram.com/ProbabilityDensityFunction.html or http://en.wikipedia.org/wiki/Probability_density_function.

${ }^{9}$ See for instance H. T. Yang et al., Appl. Phys. Lett. 94, 013103 (2009); J. C. Denardin et al., Phys. Rev. B 65, 064422 (2002); F. Wiekhorst et al., ibid. 67, 224416 (2003); S. Mitra et al., J. Magn. Magn. Mater. 306, 254 (2006); R. Zheng et al., ibid. 321, L21 (2009); T. Bitoh et al., ibid. 154, 59 (1996).

${ }^{10}$ M. El-Hilo, K. O'Grady, and R. W. Chantrell, J. Magn. Magn. Mater. 114, 295 (1992).

${ }^{11}$ The mean volume is exactly defined from the PDF $\rho(D)$ by $\bar{V}=\int_{0}^{\infty} V \rho(D) d D$; and in the same way, the mean value $\bar{X}$ of any quantity $X$ related to the particle size can be expressed as $\bar{X}=\int_{0}^{\infty} X \rho(D) d D$. The median diameter $D_{m}$ (no need to add "of the number distribution") is defined from the PDF [i.e., from $\rho(D)$ and not $f(D)$ ], by the relation: $\int_{0}^{D_{m}} \rho(D) d D=0.5$. This defines the one and only median particle diameter.

${ }^{12} \mathrm{~A}$ constant $t_{m}$ in the expression used by El-Hilo would mean that for each temperature $T$ (i.e., each point of the ZFC and FC curves), the system is brought instantly from zero temperature to $T$ and then measured after a time $t_{m}$. The system should then be demagnetized (i.e., reach the superparamagnetic regime) and cooled down (without any applied field for the ZFC curve) to zero temperature between each measurement point. This is quite unrealistic.

${ }^{13}$ Besides, note that in R. W. Chantrell et al. [IEEE Trans. Magn. 27, 3570 (1991)], this distribution is simply referred to as "the distribution of blocking temperatures," which is highly confusing because it is not the PDF. 